\title{
Optical turbulence in fiber lasers
}

\author{
Stefan Wabnitz \\ Dipartimento di Ingegneria dell'Informazione, Universita' di Brescia, Via Branze 38, \\ Brescia 25123, Italy (stefan.wabnitz@unibs.it)
}

Received December 26, 2013; accepted January 22, 2014; posted January 29, 2014 (Doc. ID 203704); published March 5, 2014

\begin{abstract}
We analyze the nonlinear stage of modulation instability in passively mode-locked fiber lasers leading to chaotic or noise-like emission. We present the phase-transition diagram among different regimes of chaotic emission in terms of the key cavity parameters: amplitude or phase turbulence, and spatio-temporal intermittency. (C) 2014 Optical Society of America

OCIS codes: (140.3510) Lasers, fiber; (190.3100) Instabilities and chaos; (190.4370) Nonlinear optics, fibers; (190.5530) Pulse propagation and temporal solitons.

http://dx.doi.org/10.1364/OL.39.001362
\end{abstract}

In addition to short-pulse and cw emission regimes, ring fiber lasers used for passive mode locking via nonlinear polarization rotation exhibit the noise-like pulse emission mode of operation [ $\underline{1}-\underline{5}]$. The resulting intense pulses have a broadband spectrum and low coherence length, which is of interest for metrology applications such as optical coherence tomography. Noise-like or turbulent laser emission may occur with both anomalous or normal cavity dispersion. Switching among various chaotic regimes involving different pulse timescales is obtained by adjusting the intracavity polarization controllers, which must be set away from the condition of minimal polarizer transmissivity for low power signals, so that weak mode-locking results. When operating in the average anomalous dispersion regime, coexistence and interaction of isolated soliton pulses with a chaotic background may lead to complex temporal dynamics involving, e.g., soliton rain and their condensation in liquid, glass, or crystal states [6,7].

In this work we point out that the dynamics of turbulent light emission from fiber lasers with passive mode locking may be analyzed by means of a universal model, namely the complex Ginzburg-Landau equation (CGLE) [8]. This approach permits us to directly relate the key cavity parameters to phase transitions among different regimes of chaotic behavior. Previous studies have been mostly devoted to the stable short-pulse generation regime, where nonlinear gain describes a fast saturable absorber [9-11]. In that case, for mode-locked soliton stability, a quintic nonlinear gain saturation term is added to the CGLE [11]. Here we analyze the modulation instability (MI) of cw emission and its associated nonlinear development and consider situations where the intracavity polarization analyzer acts as fast nonlinear gain saturation element. In this case the cubic CGLE is an appropriate minimal model.

Light propagation in a fiber laser may be described in the mean field approximation by the CGLE [12]:

$$
\begin{aligned}
t_{r} \partial_{t^{\prime}} E= & \frac{\left(g_{0} L-T^{2}\right)}{2} E+L\left(\beta_{0}+i \frac{\beta_{2}}{2}\right) \partial_{\tau^{\prime}}^{2} E \\
& -L\left(\frac{\sigma_{0}}{2}-i \gamma_{0}\right)|E|^{2} E
\end{aligned}
$$

where $t^{\prime}$ is a continuous slow temporal variable that replaces the round-trip number, $\tau^{\prime}$ is a retarded time, $E$ is the complex field envelope defined so that $|E|^{2}$ measures optical power, $t_{r}$ is the round-trip time for a cavity of length $L, g_{0}>0$ is the average distributed power gain in the cavity at the center frequency $\omega_{0}$, and $T$ is the amplitude transmission coefficient of the output coupler. Moreover, we approximated the cavity bandwidth limited gain as $G(\omega)=g_{0}\left(1-\left(4 / B^{2}\right)\left(\omega-\omega_{0}\right)^{2}\right)$, so that $\beta_{0}=4 g_{0} / B^{2}, \beta_{2}$ is the average group velocity dispersion (GVD), $\gamma_{0}=\omega_{0} n_{2} / c A_{\text {eff }}$ is the nonlinear coefficient of the fiber, $n_{2}$ is the nonlinear index, and $A_{\text {eff }}$ the effective mode area. Gain bandwidth limitation results from the amplifier response as well as from intracavity filtering, e.g., the frequency dependent polarizer transmissivity owing to fiber birefringence. With a linearly birefringent fiber, the nonlinear saturable gain coefficient is $\sigma_{0}=\gamma_{0} \cos (2 \theta) \sin (2 \theta) \sin (2 \chi) /[3(1+\cos (2 \varphi) \cos (2 \chi)$ $\cos (2 \theta)+\sin (2 \varphi) \cos (2 \chi) \sin (2 \theta))]$, where $\theta$ is the pulse input angle with respect to the fiber axes, and $(2 \chi, 2 \varphi)$ represent the angular coordinates of the polarization analyzer (which includes fiber birefringence compensation) on the Poincaré sphere [9]. Note that for a twisted or isotropic fiber, larger values of $\sigma_{0}$ may result. The sign of $\sigma_{0}$ may be changed by proper adjustment of the intracavity polarization controller: henceforth we will take $\sigma_{0}>0$.

It proves convenient to rewrite Eq. (1) in dimensionless units as

$$
\partial_{t} U=U+(1+i \beta) \partial_{\tau}^{2} U-(1-i \gamma)|U|^{2} U,
$$

where $t=\hat{g} t^{\prime} / 2 t_{r}$ : here $\hat{g}=g_{0} L-T^{2}$. Moreover, $\tau=\tau^{\prime} \sqrt{\hat{g} / 2 \beta_{0} L}, \quad U=\sqrt{\sigma_{0} L / \hat{g}} E, \quad \beta=-\beta_{2} / 2 \beta_{0}, \quad$ and $\gamma=2 \gamma_{0} / \sigma_{0}$. The $\mathrm{cw}$ solution of Eq. (2) is $U_{\mathrm{cw}}(t, \tau)=$ $\sqrt{1-\Omega^{2}} \exp \{i(K t-\Omega \tau)\}$, where $K(\Omega)=\gamma-(\gamma+\beta) \Omega^{2}$; its stability requires that $\Omega^{2}<[1-\gamma \beta] /\left[2 \gamma^{2}-\gamma \beta+3\right]$. Otherwise MI [or Benjamin-Feir (BF) instability] develops, which leads to the growth of slow temporal modulations Eq. (2) whenever $\gamma \beta>1$. The corresponding BF line $\gamma \beta=1$ is indicated by a dashed black line in Fig. 1 . Here all points above the BF line correspond to unstable chaotic solutions of the CGLE (2). Indeed, Fig. 1 provides the phase diagram of transitions from stable to intermittent and chaotic emission states of the fiber laser, as a result of the nonlinear development of MI [13].

In Fig. 1 the GVD may be anomalous (for $\beta>0$ ) or normal (for $\beta<0$ ), whereas the nonlinear coefficient $\gamma$ 


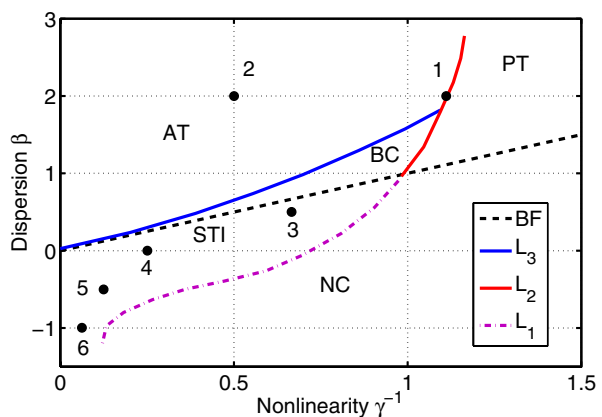

Fig. 1. Phase diagram of turbulent behavior of CGLE solutions.

is always positive. The different regions in Fig. 1, indicating various dynamical regimes of laser emission, are separated by phase transition lines. The dotted-dashed gray (magenta online) line $L_{1}$ in Fig. 1 separates the stable, nonchaotic (NC) cw emission region from a region of spatio-temporal intermittency (STI). Note that the STI domain is limited from the above by the BF line; thus it occurs in the BF or modulationally stable regime. Moreover, points to the right of the solid grey (red online) line $L_{2}$ and above the BF line in Fig. 1 correspond to the phase turbulence (PT) regime, where the irregular behavior is essentially confined to the phase evolution of the field, whereas the amplitude evolution is slaved to the phase dynamics. All points above the solid dark (blue online) line $L_{3}$ in Fig. 1 belong to the amplitude turbulence (AT) regime. Finally, the narrow domain above the BF line and between the AT and PT regions corresponds to the bi-chaos $(\mathrm{BC})$ regime, where a mixing of the AT and PT properties occurs.

Let us illustrate by selected numerical solutions of the CGLE (2) the different dynamical behaviors presented in Fig. 1. We numerically solved Eqs. (2) with the split-step Fourier method using up to $N=1024$ modes, a typical integration step $d t=10^{-3}$ and periodic boundary conditions $U\left(t, \tau=-\tau_{m} / 2\right)=U\left(t, \tau=\tau_{m} / 2\right)$. In Figs. 2(a) and 2(b) we show by contour plots the evolution of the intensity $|U|^{2}$ and phase $\arg (U)$, respectively, versus fast time $\tau$ and slow time $t$, for anomalous GVD $(\beta=2)$ and $\gamma=0.9$. This correponds to point 1 on Fig. 1 , at the boundary $L_{2}$ between the PT and the AT domains. The input condition for Eq. (2) was $U(t=0, \tau)=1+n(\tau)$, where $n(\tau)$ is a complex random noise with normal distribution, zero average, and variance $\sigma_{n}^{2}=0.001$.

As it can be seen by Fig. 2(a), in the PT regime the field amplitude exhibits irregular oscillations about the unit value, but never drops down to zero: see also Fig. 3(a), where we plot the intensity profile at $t=200$. Indeed, the corresponding power spectrum of Fig. 3(b) shows that the dominant $\mathrm{cw}$ component is about $20 \mathrm{~dB}$ stronger that the spectral wings. The phase evolution of the field in Fig. 2(b) shows that in the PT regime irregular phase jumps may occur.

It is quite remarkable that in proximity of the $\mathrm{BF}$ line in the phase diagram of Fig. 1, one may get different reduced descriptions of the wave evolution which, while preserving the chaotic behavior, may lead to analytical insight in terms of exact solutions, which act as dynamical attractors. In fact, we may write a perturbed cw
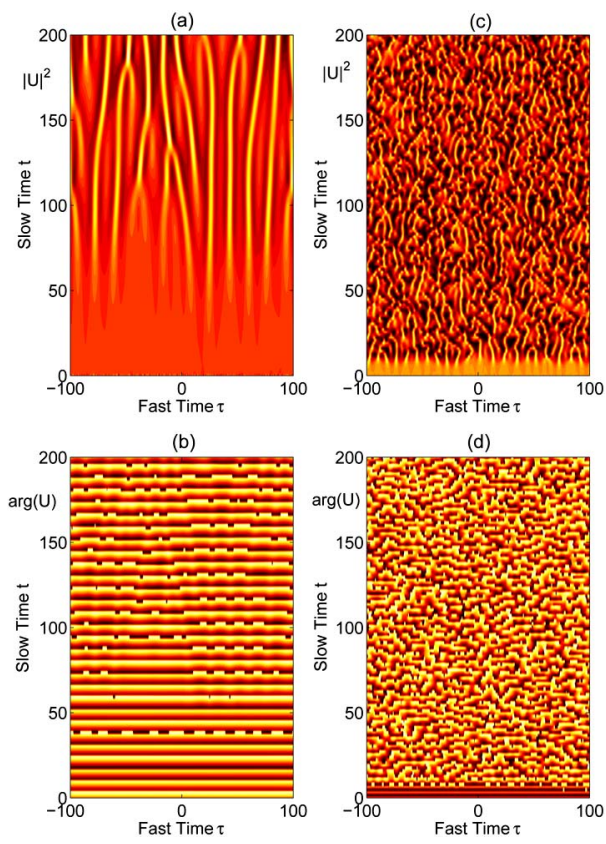

Fig. 2. Contour plots showing the evolution of the (a), (c) intensity $|U(t, \tau)|^{2}$ and (b), (d) phase $\arg (U)$ versus fast time $\tau$ and slow time $t$ : (a), (b) $\beta=2, \gamma=0.9$ (PT regime, point 1 in Fig. 1); (c), (d) $\beta=2, \gamma=2$ (AT regime, point 2 in Fig. 1).

solution of Eq. (2) as $U(t, \tau)=U_{0}(1+u) \exp (i \theta)$ where $U_{0}$ is the cw solution with $\Omega=0$. By inserting such ansatz in the CGLE (2), one may write down two evolution equations for the amplitude $u$ and the phase $\theta$. By supposing a slow $\tau$-variation of $u$ and $\theta$, namely that $\partial_{\tau} \approx \varepsilon$, where $\varepsilon \ll 1$, it turns out that $\partial_{t} \approx \varepsilon^{2}, u \approx \varepsilon^{2}$, and $\theta \approx \varepsilon^{0}[14]$. By keeping only the dominant terms in the equations for $u$ and $\theta$, one obtains that the chirp $\nu \equiv$ $\partial_{\tau} \theta$ obeys the hydrodynamic Burgers equation,

$$
\partial_{t} \nu=\eta \partial_{\tau}^{2} \nu-\lambda \nu \partial_{\tau} \nu
$$

where $\eta=1-\beta \gamma$ and $\lambda=2(\beta+\gamma)$. Whereas the amplitude $u \cong-\left\{\beta \partial_{\tau}^{2} \theta+\left(\partial_{\tau} \theta\right)^{2}\right\} / 2$ is slaved to the phase evolution. For a stable dissipative balance between gain and loss terms, it is necessary that $\eta>0$ in Eq. (3), which therefore only applies in regions below the $\mathrm{BF}$ line in Fig. 1. The Burgers equation (3) is a well known model for one-dimensional turbulence in hydrodynamics and chemical reactions.

Conversely, above the BF line (for example in the PT regime) where $\eta<0$, to ensure a stable balance among gain and loss, it is necessary to retain an additional higher-order viscous damping or dissipative term (i.e., the fourth-order temporal derivative of the phase $\theta$ ) from the amplitude and phase equations. For small values of $|\eta|$, one obtains the Kuramoto-Sivashinsky equation (KSE) for the chirp $\nu$ :

$$
\partial_{t} \nu=-|\eta| \partial_{\tau}^{2} \nu-\mu \partial_{\tau}^{4} \nu-\lambda \nu \partial_{\tau} \nu,
$$

where $\mu=\left(1+\beta^{2}\right) / 2$. The KSE also models instabilities on laminar flame fronts, PT in chemical oscillations, and phase dynamics in reaction-diffusion systems. The first (second) linear term on the right-hand side of Eq. ( $\underline{4}$ 

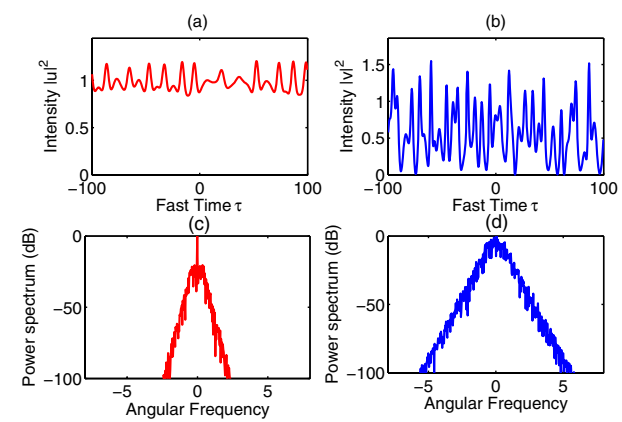

Fig. 3. Intensity $|U(t=200, \tau)|^{2}$ and power spectrum for (a), (b) $\beta=2, \gamma=0.9$ (PT regime); (c), (d) $\beta=2, \gamma=2$ (AT regime).

leads to instability (damping) at low (high) frequencies; whereas the third nonlinear term has a stabilizing effect by dynamically transferring energy from low to high frequencies. A relatively simple solution of the KSE Eq. (4) is provided by a bound pair of shock waves of the form $\nu=\nu_{0} \tilde{\nu}\left(\tau / \tau_{0}\right)$, where

$$
\tilde{\nu}(x)=\left(-60 \rho^{3}+\frac{30}{19} \rho\right) \tanh (\rho x)+60 \rho^{3} \tanh ^{3}(\rho x),
$$

with $\nu_{0}=\lambda^{-1} \sqrt{|\eta|^{3} / \mu}, \tau_{0}=\sqrt{\mu /|\eta|}$, and $\rho=\sqrt{209} / 38$ [14]. Equation (5) describes a period of oscillation of the chirp $\nu$ that connects a jump among two different frequency values. Indeed, Fig. $\underline{4}$ shows a train of shock pairs which is generated at $t=200$ in correspondence of the PT state described by point 1 in Fig. 1 . Here we numerically solved Eq. (4) with the input condition $\nu(t=0, \tau)=\exp \left\{-(\tau-10)^{2}\right\}-\exp \left\{-(\tau+10)^{2}\right\}$.

In sharp contrast, Figs. 2(c) and 2(d) show that highly irregular amplitude and phase evolutions occur in the AT regime: here $\beta=2$ and $\gamma=2$ (point 2 on Fig. 1). The corresponding intensity profile in Fig. 3(c) at $t=200$ shows irregular, large amplitude oscillations between the extreme values of 0 and 1.5. Inspection of Fig. 2(c) reveals that in the AT regime the chaotic behavior is associated with the random appearance of defects (dark points), separated by narrow bright stripes. The associated power spectrum of Fig. 3(d) shows significant spectral broadening with respect to the PT case of Fig. 3(b).

Figure $\underline{5}$ shows two different examples of field intensity and phase evolutions in the STI regime. In Figs. 5(a) and 5(b) the GVD is anomalous $\beta=0.5$ and $\gamma=1.5$ (point 3 on Fig. 1). Whereas in Figs. 5(c) and 5(d) the GVD $\beta=0$ and $\gamma=\overline{4}$ (point 4 on Fig. 1). In the STI simulations, we

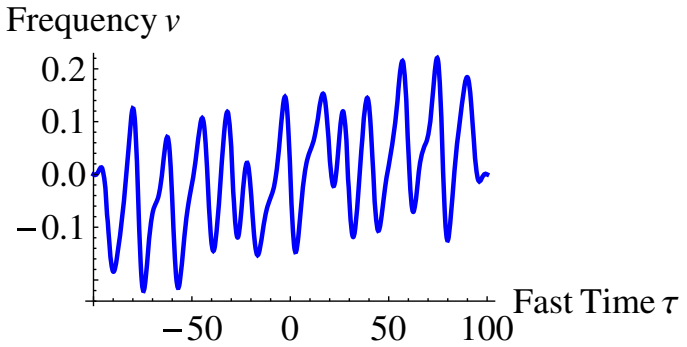

Fig. 4. Frequency chirp $\nu(t=200, \tau)$ from the KSE (4) for the PT solution of point 1 in Fig. 1 .
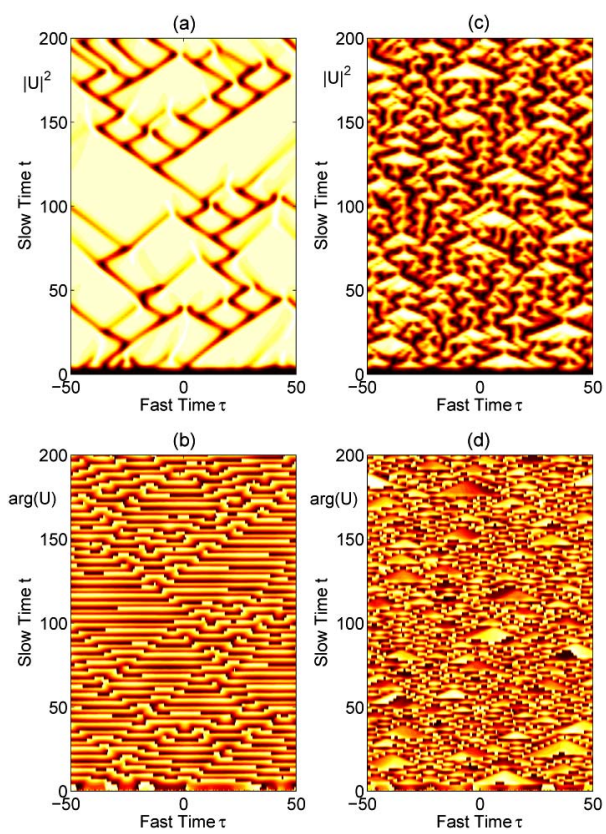

Fig. 5. As in Fig. 2 in the STI regime with: (a), (b) $\beta=0.5, \gamma=$ 1.5 (point 3 in Fig. 1 ); (c), (d) $\beta=0, \gamma=4$ (point 4 in Fig. 1 ).

set as an input condition for Eq. (2) $U(t=0, \tau)=n(\tau)$, where the complex noise variance $\sigma_{n}^{2}=0.01$. In the first case of Fig. 5(a), symmetric diamond shaped patches of cws (bright laminar regions) are separated by propagating shocks and dark holes, which corresponds to homoclinic trajectories generated at points of zero amplitude, where a phase slip occurs, as shown by Fig. 5(b). The corresponding shapshot intensity profile in Fig. 6(a) shows two holes separated by a $\mathrm{cw}$ domain containing a shock.

Note that the spectral bandwidth in the STI regime [see Fig. 6(b)] is reduced with respect to the AT regime [Fig. $3(\mathrm{~d})]$. Figures $\underline{5(\mathrm{c})}$ and $5(\mathrm{~d})$ show that as the average dispersion touches zero the spatio-temporal dimension of the $\mathrm{cw}$ regions is substantially contracted, and their shape acquires an asymmetric triangular pattern. The corresponding output power profile in Fig. 6(c) shows that significant merging of the holes has occurred, which increases the spectral broadening as seen in Fig. $6(d)$.

The phase diagram of Fig. 1 shows that the STI domain extends into the normal GVD regime $(\beta<0)$. The plots in Figs. 7(a) and 7(b) show the irregular intensity evolutions corresponding to points 5 and 6 in Fig. 1 , respectively.
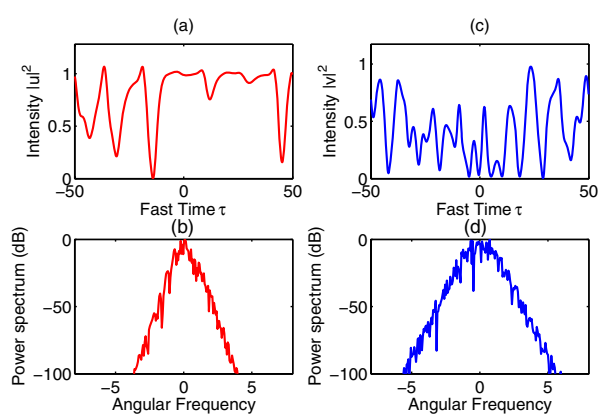

Fig. 6. As in Fig. $\underline{3}$, in the STI regime with (a), (b) $\beta=0.5$, $\gamma=1.5 ;$ (c), (d) $\beta=0, \gamma=4$. 

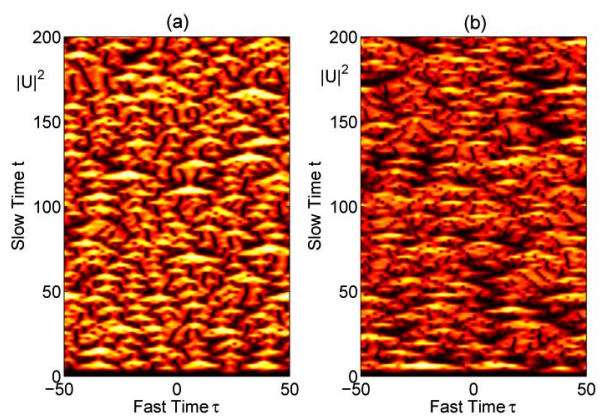

Fig. 7. As in Fig. 2(a) in STI regime with (a), (b) $\beta=-0.5, \gamma=$ 8 (point 5 in Fig. 1) (c), (d) $\beta=-1, \gamma=16$ (point 6 in Fig. 1).

The associated intensity profiles and power spectra at $t=200$ (not shown here) reveal that using a cavity with normal GVD leads to output spectra with significantly broader bandwidths than in the anomalous GVD regime.

Useful information on the time scale of coherent structures within the turbulent patterns is provided by intensity autocorrelations. Figure 8(a) compares the PT and the AT regimes of points 1 and 2, respectively. As can be seen, the relatively shallow fluctuations on a background of the PT regime lead to a nearly triangular, broad autocorrelation, whereas the narrow autocorrelation peak of the AT regime measures the duration of intensity defects. The autocorrelations of Fig. $8(\mathrm{~b})$ for the STI regime show that the duration of the central peak remains nearly unchanged for either zero or normal GVD (points $4,5,6)$, whereas its grows significantly larger in the anomalous GVD regime (point 3).

In conclusion, we presented a universal model for describing regimes of turbulent behavior in passively mode locked fiber lasers. This study provides a framework for the design of broadband, low-coherence and high-power pulsed light sources. Further developments may involve the analysis of interactions between solitons and a cw [15], and dissipative rogue wave generation [16].

This research was funded by the Italian Ministry of University and Research (grant no. 2012BFNWZ2), and Fondazione Cariplo (grant no. 2011-0395).
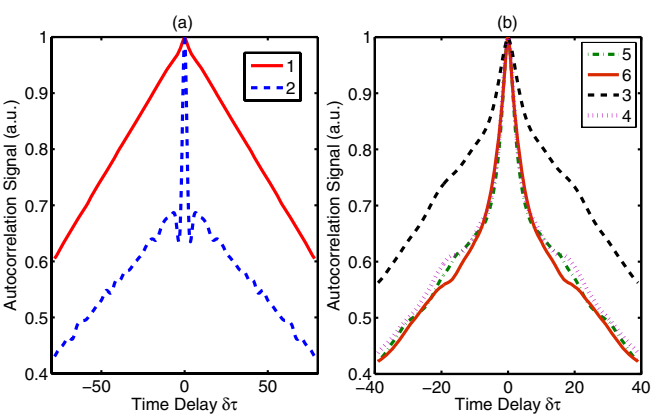

Fig. 8. Numerical intensity autocorrelation traces. Labels indicate corresponding points in Fig. 1 .

\section{References}

1. M. Horowitz, Y. Barad, and Y. Silberberg, Opt. Lett. 22, 799 (1997).

2. M. Horowitz and Y. Silberberg, Phot. Techn. Lett. 10, 1389 (1998).

3. Y. Takushima, K. Yasunaka, Y. Ozeki, and K. Kikuchi, Electron Lett. 41, 399 (2005).

4. S. Smirnov, S. Kobtsev, S. Kukarin, and A. Ivanenko, Opt. Express 20, 27447 (2012).

5. A. F. Runge, C. Aguergaray, N. G. R. Broderick, and M. Erkintalo, Opt. Lett. 38, 4327 (2013).

6. S. Chouli and Ph. Grelu, Phys. Rev. A 81, 063829 (2010).

7. F. Amrani, A. Haboucha, M. Salhi, H. Leblond, A. Komarov, and F. Sanchez, Appl. Phys. B 99, 107 (2010).

8. B. I. Shraiman, A. Pumir, W. van Saarloos, P. C. Hoenberg, H. Chaté, and M. Holen, Phys. D 57, 241 (1992).

9. Y. Kodama, M. Romagnoli, and S. Wabnitz, Electron. Lett. 28, 1981 (1992).

10. A. I. Chernykh and S. K. Turitsyn, Opt. Lett. 20, 398 (1995).

11. Ph. Grelu and N. Akhmediev, Nat. Photonics 6, 84 (2012).

12. M. Haelterman, S. Trillo, and S. Wabnitz, Opt. Commun. 91, 401 (1992).

13. H. Chaté, Nonlinearity 7, 185 (1994).

14. Y. Kuramoto and T. Tsuzuki, Prog. Theor. Phys. 55, 356 (1976).

15. H. Leblond, A. Niang, F. Amrani, M. Salhi, and F. Sanchez, Phys. Rev. A 88, 033809 (2013).

16. C. Lecaplain, Ph. Grelu, J. M. Soto-Crespo, and N. Akhmediev, Phys. Rev. Lett. 108, 233901 (2012). 